\title{
Mixed methods approach and its implications in acute care research
}

Asim $\mathrm{M}^{1 *}$, Mekkodathil $\mathrm{A}^{2}$, Ibnas $\mathrm{M}^{3}$, Sathian $\mathrm{B}^{4}$

*Corresponding author:

${ }^{1}$ Dr. Mohammad Asim, Ph.D, Academic Research Associate, Trauma Surgery, Department of Surgery, Hamad General Hospital, Doha, Qatar.

Email: asim.jmi@gmail.com ORCID

${ }^{2}$ Injury prevention coordinator, Clinical Research, Trauma Surgery, Surgery Department, Hamad General Hospital, Doha, Qatar.

${ }^{3}$ Research Coordinator, Clinical Research, Trauma Surgery, Surgery Department, Hamad General Hospital, Doha, Qatar.

${ }^{4}$ Academic Research Associate, Clinical Research, Trauma Surgery, Surgery Department, Hamad General Hospital, Doha, Qatar

Information about the article:

Received: Jan. 17, 2019

Accepted: Feb. 20, 2019

Published online: Dec. 27, 2019

\section{Publisher}

Nepal Health Research Society, Bahundhara -6, Gokarnesowor Municipality, Kathmandu, Nepal

eISSN 2382-5545, ISSN 2676-1343 (Print)

(C) The Author(s). 2019

Content licensing: CC BY 4.0

\begin{abstract}
\section{Background}

The acute care settings have the potential to facilitate robust epidemiological and public health research necessary for evidence-based system. The methods in emergency research are well suited for mixed methods research. Despite the use of quantitative and qualitative data in emergency settings, mixed methods studies in this field are limited to help researchers to address health care issues. Therefore, we aim to assess the prospects and implications of mixed methods approach in acute care settings.
\end{abstract}

\section{Materials and Methods}

We have reviewed the published English literature using the research databases (PubMed, Medline, CINHAL and Google Scholar) related to the implications of mixed methods in emergency research, in order to understand and broaden the scope of patient care in time critical conditions.

\section{Results}

Basically, there are four mixed method approaches which integrate qualitative and quantitative methods (such as Triangulation, Embedded, Explanatory and Exploratory design) which can be selected depending upon the research question. In a single study, appropriate selection of mixed method approach enables quality research which involves understanding of the different aspects of human interaction and paradigms to characterize complex healthcare systems from societal standpoint. So, the combination approach may answer key issues in emergency settings which enriches and enhances the healthcare practice that may influence patient outcomes. Notably, emergency research has wider range of parameters which not only benefit the patients but also help in overcoming system-related clinical challenges. Of note, the ethical conduct of study is crucial in a complex emergency situation which remains challenging for recruitment and obtaining informed consent from subject or their nest of kin. Another impediment is the lack of trained emergency researcher to support the research activities and data collection in the emergent settings.

\section{Conclusion}

Application of mixed approach is of great importance in emergency medicine to enhance our understanding of critical care research that may add valuable insight on various aspects of human behavior. Therefore, appropriate planning and integration of study design may help researchers to explore newer avenues to improve patients care in emergency settings.

\section{Keywords}

Mixed methods approach, scope, application, emergency settings 